\title{
Fluid State of Dirac Quantum Particles
}

\author{
Vu B. Ho \\ Advanced Study, 9 Adela Court, Mulgrave, Victoria, Australia \\ Email: vubho@bigpond.net.au
}

How to cite this paper: Ho, V.B. (2018) Fluid State of Dirac Quantum Particles. Journal of Modern Physics, 9, 2402-2419. https://doi.org/10.4236/jmp.2018.914154

Received: November 7, 2018

Accepted: December 10, 2018

Published: December 13, 2018

Copyright $\odot 2018$ by author and Scientific Research Publishing Inc. This work is licensed under the Creative Commons Attribution International License (CC BY 4.0).

http://creativecommons.org/licenses/by/4.0/

(c) (i) Open Access

\begin{abstract}
In our previous works, we suggest that quantum particles are composite physical objects endowed with the geometric and topological structures of their corresponding differentiable manifolds that would allow them to imitate and adapt to physical environments. In this work, we show that Dirac equation in fact describes quantum particles as composite structures that are in a fluid state in which the components of the wavefunction can be identified with the stream function and the velocity potential of a potential flow formulated in the theory of classical fluids. We also show that Dirac quantum particles can manifest as standing waves which are the result of the superposition of two fluid flows moving in opposite directions. However, for a steady motion a Dirac quantum particle does not exhibit a wave motion even though it has the potential to establish a wave within its physical structure, therefore, without an external disturbance a Dirac quantum particle may be considered as a classical particle defined in classical physics. And furthermore, from the fact that there are two identical fluid flows in opposite directions within their physical structures, the fluid state model of Dirac quantum particles can be used to explain why fermions are spin-half particles.
\end{abstract}

\section{Keywords}

Dirac Equation, Wave Mechanics, Stan, Fluid Mechanics, Stream Function, Velocity Potential, Potential Flow, General Relativity, Maxwell Field Equations, CW Complexes, Differential Geometry, Topology, Differentiable Manifolds, Topological Transformation

\section{Introductory Summary}

In our previous works on spacetime structures of quantum particles, we suggest that quantum particles should be endowed with geometric and topological structures of differentiable manifolds and their motion should be described as 
isometric embeddings in higher Euclidean space. We also suggest that all quantum particles are formed from mass points which are joined together by contact forces as a consequence of viewing quantum particles as CW-complexes [1] [2] [3]. Fundamentally, we show that the three main dynamical descriptions of physical events in classical physics, namely Newton mechanics, Maxwell electromagnetism and Einstein gravitation, can be formulated in the same general covariant form and they can be represented by the general equation

$$
\nabla_{\beta} M=k J
$$

where $M$ is a mathematical object that represents the corresponding physical system and $\nabla_{\beta}$ is a covariant derivative. For Newton mechanics, $M=\frac{1}{2} m \sum_{\mu=1}^{3}\left(\mathrm{~d} x^{\mu} / \mathrm{d} t\right)^{2}+V$ and $J=0$. For Maxwell electromagnetism, $M=F^{\alpha \beta}=\partial^{\mu} A^{v}-\partial^{v} A^{\mu}$, with the four-vector potential $A^{\mu} \equiv(V, \mathbf{A})$ and $J$ can be identified with the electric and magnetic currents. And for Einstein gravitation, $M=R^{\alpha \beta}$ and $J$ can be defined in terms of a metric $g_{\alpha \beta}$ and the Ricci scalar curvature using the Bianchi identities $\nabla_{\beta} R^{\alpha \beta}=\frac{1}{2} g^{\alpha \beta} \nabla_{\beta} R$, that is, $J=\frac{1}{2} g^{\alpha \beta} \nabla_{\beta} R$. If we use the Bianchi identities as field equations for the gravitational field then Einstein field equations $T_{\mu v}=k\left(R_{\mu v}-\frac{1}{2} R g_{\mu v}+\Lambda g_{\mu v}\right)$, as in the case of the electromagnetic field, should be regarded as a definition for the energy-momentum tensor $T_{\mu \nu}$ for the gravitational field [4]. An interesting feature that emerges from Equation (1) for the gravitational field is that we can derive the Ricci flow $\partial g_{\alpha \beta} / \partial t=\kappa R_{\alpha \beta}$ for a vacuum field $J=0$. Mathematically, the Ricci flow is a geometric process that can be employed to smooth out irregularities of a Riemannian manifold [5]. From the definition of the four-current $j^{\alpha}=\left(\rho, \mathbf{j}_{i}\right)=\frac{1}{2} g^{\alpha \beta} \nabla_{\beta} R$ for the gravitational field, by comparing with the Poisson equation for a potential $V$ in classical physics, $\nabla^{2} V=4 \pi \rho$, we can identify the scalar potential $V$ with the Ricci scalar curvature $R$ and then obtain a diffusion equation $\partial_{t} R=k \nabla^{2} R$ whose solutions can be found to take the form $R(x, y, z, t)=\left(M /(\sqrt{4 \pi k t})^{3}\right) \mathrm{e}^{-\left(x^{2}+y^{2}+z^{2}\right) / 4 k t}$, which determines the probabilistic distribution of an amount of geometrical substance $M$ which is defined via the Ricci scalar curvature $R$ and manifests as observable matter [6]. It is worth mentioning that in fact a similar diffusion equation can also be derived from the Ricci flow $\partial g_{\alpha \beta} / \partial t=\kappa R_{\alpha \beta}$ of the form $\partial R / \partial t=\Delta R+2 \mid$ Ric $\left.\right|^{2}$, where $\Delta$ is the Laplacian defined as $\Delta=g^{\alpha \beta} \nabla_{\alpha} \nabla_{\beta}$ and $\mid$ Ric $\mid$ is a shorthand for a mathematical expression that we will not be concerned with in this work [7]. Therefore, the Bianchi field equations of general relativity in the covariant form given in Equation (1) can be used to formulate quantum particles as differentiable manifolds. For example, we showed that the Ricci scalar curvature $R$ associated with a differentiable manifold that represents a quantum system, such as the hydrogen atom, can be expressed in terms of the Schrödinger wavefunction $\psi$ in quantum mechanics as

$$
R=k\left(\sum_{\mu=1}^{3}\left(\mathrm{~d} x^{\mu} / \mathrm{d} t\right)^{2}-(\hbar / m)\left(\partial_{t} \psi+\sum_{\mu=1}^{3} \partial_{\mu} \psi\left(\mathrm{d} x^{\mu} / \mathrm{d} t\right) / \psi\right)\right) \text {. }
$$


On the other hand, we have also shown that Maxwell field equations of electromagnetism and Dirac relativistic equation of quantum mechanics can be formulated covariantly from a general system of linear first order partial differential equations [8] [9]. An explicit form of a system of linear first order partial differential equations can be written as follows [10] [11]

$$
\sum_{i=1}^{n} \sum_{j=1}^{n} a_{i j}^{r} \frac{\partial \psi_{i}}{\partial x_{j}}=k_{1} \sum_{l=1}^{n} b_{l}^{r} \psi_{l}+k_{2} c^{r}, r=1,2, \cdots, n
$$

The system of equations given in Equation (2) can be rewritten in a matrix form as

$$
\left(\sum_{i=1}^{n} A_{i} \frac{\partial}{\partial x_{i}}\right) \psi=k_{1} \sigma \psi+k_{2} J
$$

where $\psi=\left(\psi_{1}, \psi_{2}, \cdots, \psi_{n}\right)^{\mathrm{T}}, \quad \partial \psi / \partial x_{i}=\left(\partial \psi_{1} / \partial x_{i}, \partial \psi_{2} / \partial x_{i}, \cdots, \partial \psi_{n} / \partial x_{i}\right)^{\mathrm{T}}, \quad A_{i}$, $\sigma$ and $J$ are matrices representing the quantities $a_{i j}^{k}, b_{l}^{r}$ and $c^{r}$, and $k_{1}$ and $k_{2}$ are undetermined constants. Now, if we apply the operator $\sum_{i=1}^{n} A_{i} \partial / \partial x_{i}$ on the left on both sides of Equation (3) then we obtain

$$
\begin{aligned}
& \left(\sum_{i=1}^{n} A_{i}^{2} \frac{\partial^{2}}{\partial x_{i}^{2}}+\sum_{i=1}^{n} \sum_{j>i}^{n}\left(A_{i} A_{j}+A_{j} A_{i}\right) \frac{\partial^{2}}{\partial x_{i} \partial x_{j}}\right) \psi \\
& =k_{1}^{2} \sigma^{2} \psi+k_{1} k_{2} \sigma J+k_{2} \sum_{i=1}^{n} A_{i} \frac{\partial J}{\partial x_{i}}
\end{aligned}
$$

In order for the above systems of partial differential equations to be used to describe physical phenomena, the matrices $A_{i}$ must be determined. We have shown that for both Dirac and Maxwell field equations, the matrices $A_{i}$ must take a form so that Equation (4) reduces to the following equation

$$
\left(\sum_{i=1}^{n} A_{i}^{2} \frac{\partial^{2}}{\partial x_{i}^{2}}\right) \psi=k_{1}^{2} \sigma^{2} \psi+k_{1} k_{2} \sigma J+k_{2} \sum_{i=1}^{n} A_{i} \frac{\partial J}{\partial x_{i}}
$$

To obtain Dirac equation we simply set $A_{i} A_{j}+A_{j} A_{i}=0$ with $A_{i}^{2}= \pm 1$, and in this case the matrices $A_{i}$ are the matrices $\gamma_{i}$ given as [12]

$$
\begin{aligned}
& \gamma_{1}=\left(\begin{array}{cccc}
1 & 0 & 0 & 0 \\
0 & 1 & 0 & 0 \\
0 & 0 & -1 & 0 \\
0 & 0 & 0 & -1
\end{array}\right), \gamma_{2}=\left(\begin{array}{cccc}
0 & 0 & 0 & 1 \\
0 & 0 & 1 & 0 \\
0 & -1 & 0 & 0 \\
-1 & 0 & 0 & 0
\end{array}\right), \\
& \gamma_{3}=\left(\begin{array}{cccc}
0 & 0 & 0 & -i \\
0 & 0 & i & 0 \\
0 & i & 0 & 0 \\
-i & 0 & 0 & 0
\end{array}\right), \gamma_{4}=\left(\begin{array}{cccc}
0 & 0 & 1 & 0 \\
0 & 0 & 0 & -1 \\
-1 & 0 & 0 & 0 \\
0 & 1 & 0 & 0
\end{array}\right)
\end{aligned}
$$

For Maxwell field equations, in order to specify the matrices $A_{i}$ we need to use the form of Maxwell field equations established in classical electrodynamics [13] [14]. And the matrices $A_{i}$ take the forms 


$$
\begin{aligned}
& A_{1}=\left(\begin{array}{cccccc}
-1 & 0 & 0 & 0 & 0 & 0 \\
0 & -1 & 0 & 0 & 0 & 0 \\
0 & 0 & -1 & 0 & 0 & 0 \\
0 & 0 & 0 & 1 & 0 & 0 \\
0 & 0 & 0 & 0 & 1 & 0 \\
0 & 0 & 0 & 0 & 0 & 1
\end{array}\right), A_{2}=\left(\begin{array}{cccccc}
0 & 0 & 0 & 0 & 0 & 0 \\
0 & 0 & 0 & 0 & 0 & -1 \\
0 & 0 & 0 & 0 & 1 & 0 \\
0 & 0 & 0 & 0 & 0 & 0 \\
0 & 0 & -1 & 0 & 0 & 0 \\
0 & 1 & 0 & 0 & 0 & 0
\end{array}\right), \\
& A_{3}=\left(\begin{array}{cccccc}
0 & 0 & 0 & 0 & 0 & 1 \\
0 & 0 & 0 & 0 & 0 & 0 \\
0 & 0 & 0 & -1 & 0 & 0 \\
0 & 0 & 1 & 0 & 0 & 0 \\
0 & 0 & 0 & 0 & 0 & 0 \\
-1 & 0 & 0 & 0 & 0 & 0
\end{array}\right), A_{4}=\left(\begin{array}{cccccc}
0 & 0 & 0 & 0 & -1 & 0 \\
0 & 0 & 0 & 1 & 0 & 0 \\
0 & 0 & 0 & 0 & 0 & 0 \\
0 & -1 & 0 & 0 & 0 & 0 \\
1 & 0 & 0 & 0 & 0 & 0 \\
0 & 0 & 0 & 0 & 0 & 0
\end{array}\right), \\
& A_{5}=\left(\begin{array}{llllll}
\mu & 0 & 0 & 0 & 0 & 0 \\
0 & \mu & 0 & 0 & 0 & 0 \\
0 & 0 & \mu & 0 & 0 & 0 \\
0 & 0 & 0 & 1 & 0 & 0 \\
0 & 0 & 0 & 0 & 1 & 0 \\
0 & 0 & 0 & 0 & 0 & 1
\end{array}\right)
\end{aligned}
$$

Besides the covariant formulations of classical and quantum physics as described above, we have also discussed the topological transformation of quantum dynamics by showing the wave dynamics of a quantum particle on different types of topological structures in various dimensions from the fundamental polygons of the corresponding universal covering spaces [15]. We presented our discussions in the form of Bohr model in one, two and three dimensions using linear wave equations. For the clarity of our presentation in terms of Bohr model, we want to mention here that in order to successfully construct a model for the hydrogen atom which predicts correctly the spectrum of the energy radiated from the atom, Bohr proposed three postulates which state that the centripetal force required for the electron to orbit the nucleus in a stable circle is the Coulomb force $m v^{2} / r=k q^{2} / r^{2}$, the permissible orbits are those that satisfy the condition that the angular momentum of the electron equals $n \hbar$, that is $m v r=n \hbar$, and when the electron moves in one of the stable orbits it does not radiate, however, it will radiate when it makes a transition between the stable orbits [16]. Furthermore, in his work on the concept of matter wave, de Broglie proposed that an electron has both a wave and a particle nature by regarding the electron as a standing wave around the circumference of an orbit, as shown in Figure 1 [17].

It is seen that de Broglie's requirement leads to the wave condition $2 \pi r=n \lambda$. This is equivalent to assuming that the standing wave around a circle, which is a 1 -sphere, is similar to a standing wave on the fundamental interval of a straight line which is the universal covering space of the circle $S^{1}$, where the translations taking the interval to the next images will generate the holonomy group [18]. If we apply de Broglie wavelength $\lambda$ defined in terms of the momentum 


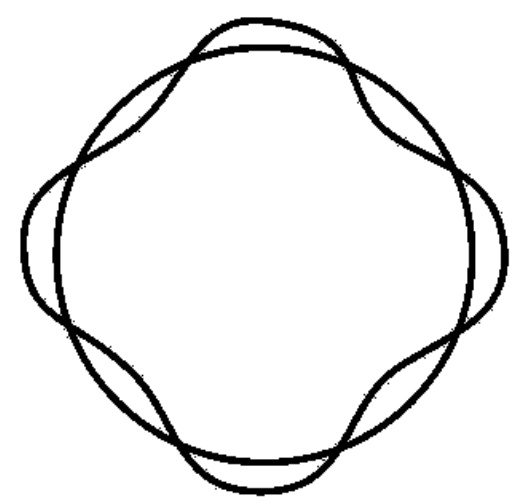

Figure 1. de Broglie waves around a circle.

of a quantum particle $p=m v$ as $\lambda=h / m v$, then using the wavelength also given by $\lambda=2 \pi R / n$ we obtain $h / m v=2 \pi R / n$, that leads to the Bohr's postulate of the quantisation of angular momentum $m v R=n \hbar$. Then the energy spectrum $E_{n}$ can be calculated from Coulomb's law $m v^{2} / R=k q^{2} / R^{2}$ as $E_{n}=-m k^{2} q^{4} / 2 \hbar^{2} n^{2}$, where $R$ now is the radius of the $n$th stationary orbit.

In mathematics, the fundamental polygon in one dimension is an interval and the universal covering space is the straight line and in this case the standing wave on a finite string is transformed into the standing wave on a circle which can be applied into the Bohr model of the hydrogen atom. In two dimensions, the fundamental polygon is a square and the universal covering space is the plane and in this case the standing wave on the square is transformed into the standing wave on different surfaces that can be formed by gluing opposite sides of the square, which include a 2-sphere, a 2-torus, a Klein bottle and a projective plane. This may be seen as an extension of the Bohr model of the hydrogen atom from one-dimensional manifolds of the 1-sphere and 1-torus embedded in the ambient two-dimensional Euclidean space $R^{2}$ into two-dimensional manifolds embedded or immersed in the ambient three-dimensional Euclidean space $R^{3}$. In three dimensions, the fundamental polygon is a cube and the universal covering space is the three-dimensional Euclidean space. It is shown that a 3-torus and the manifold $K \times S^{1}$ defined as the product of a Klein bottle and a circle can be constructed by gluing opposite faces of a cube therefore in three-dimensions the standing wave on a cube is transformed into the standing wave on a 3-torus or on the manifold $K \times S^{1}$. We also discuss a transformation of a stationary wave on the fundamental cube into a stationary wave on a 3-sphere despite it still remains unknown whether a 3 -sphere can be constructed directly from a cube by gluing its opposite faces. In spite of this uncertainty, however, we speculate that mathematical degeneracy in which an element of a class of objects degenerates into an element of a different but simpler class may play an important role in quantum dynamics. For example, a 2-sphere is a degenerate 2-torus when the axis of revolution passes through the centre of the generating circle. Therefore, it seems reasonable to assume that if an $n$-torus degenerates into an $n$-sphere then wavefunctions on an $n$-torus may also be degenerated into wavefunctions on an 
$n$-sphere. Furthermore, since an $n$-sphere can degenerate itself into a single point, therefore the mathematical degeneracy may be related to the concept of wavefunction collapse in quantum mechanics where the classical observables such as position and momentum can only be obtained from the collapse of the associated wavefunctions for physical measurements. This consideration suggests that quantum particles associated with differentiable manifolds may possess the more stable mathematical structures of an $n$-torus rather than those of an $n$-sphere.

The above formulation of quantum particles in terms of differentiable manifolds and the consideration of their intrinsic geometric and topological characteristics raise the question of how the standing waves that represent quantum particles could be established physically. The aim of this work is to answer this question by showing that Dirac equation in fact describes quantum particles as composite structures that are in a fluid state in which the components of the wavefunction can be identified with the stream function and the velocity potential of a potential flow formulated in the theory of classical fluids. In this case Dirac quantum particles can manifest as standing waves which are the result of the superposition of two fluid flows moving in opposite directions. For example, if two opposite waves are represented by the function $\psi_{1}(r, t)=a \sin (k r-v t)$ and $\psi_{2}(r, t)=a \sin (k r+v t)$ then the resultant standing wave can be obtained as $\psi_{1}(r, t)+\psi_{2}(r, t)=2 a \sin (k r) \cos (v t)$. We also show that even though a Dirac quantum particle has the potential to transfer energy in opposite directions to establish a standing wave within its physical structure, for a steady motion without an external disturbance a Dirac quantum particle can be considered as a classical particle defined in classical physics.

\section{Two-Dimensional Hydrogen-Like Physical System}

In this work we will show that quantum particles can be described as physical systems in a state of fluids that can be formulated in terms of the fluid dynamics in two dimensions. Since such systems can be seen to have the physical structure of a two-dimensional hydrogen-like atom therefore in this section we will examine further how they can be described in terms of quantum mechanics. First we need to extend our formulation of Maxwell field equations of electromagnetism and Dirac relativistic equation from a general system of linear first order partial differential equations to that with an external field. Such system of equations is given as follows [19]

$$
\sum_{i=1}^{n} \sum_{j=1}^{n} a_{i j}^{r} \frac{\partial \psi_{i}}{\partial x_{j}}=\sum_{i=1}^{n}\left(\sum_{j=1}^{n} b_{i j}^{r} V_{j}+c_{i}^{r}\right) \psi_{i}+d^{r}, r=1,2, \cdots, n
$$

The system of equations given in Equation (8) can be rewritten in a matrix form as

$$
\left(\sum_{i=1}^{n} A_{i} \frac{\partial}{\partial x_{i}}\right) \psi=-i\left(\sum_{i=1}^{n} q B_{i} V_{i}+m \sigma\right) \psi+J
$$


where $\psi=\left(\psi_{1}, \psi_{2}, \cdots, \psi_{n}\right)^{\mathrm{T}}, \quad \partial \psi / \partial x_{i}=\left(\partial \psi_{1} / \partial x_{i}, \partial \psi_{2} / \partial x_{i}, \cdots, \partial \psi_{n} / \partial x_{i}\right)^{\mathrm{T}}$ with $A_{i}, B_{i}, \sigma$ and $J$ are matrices representing the quantities $a_{i j}^{r}, b_{i j}^{r}, c_{j}^{r}$ and $d^{r}$, which are assumed to be constant in this work. While the quantities $q, m$ and $J$ represent physical entities related directly to the physical properties of the particle, and the quantities $V_{i}$ represent an external field, such as the potentials of an electromagnetic field. By applying the operator $\sum_{i=1}^{n} A_{i} \partial / \partial x_{i}$ on the left on both sides of Equation (9), with the assumption that the coefficients $a_{i j}^{k}, b_{i}^{r}$ and $c^{r}$ are constants, then we obtain

$$
\left(\sum_{i=1}^{n} A_{i} \frac{\partial}{\partial x_{i}}\right)\left(\sum_{j=1}^{n} A_{j} \frac{\partial}{\partial x_{j}}\right) \psi=\left(\sum_{i=1}^{n} A_{i} \frac{\partial}{\partial x_{i}}\right)\left(-i\left(\sum_{j=1}^{n} q B_{j} V_{j}+m \sigma\right) \psi+J\right)
$$

Since the quantities $A_{i}, B_{i}, \sigma, q, m$ and $J$ are assumed to be constant, Equation (10) becomes

$$
\begin{aligned}
& \left(\sum_{i=1}^{n} A_{i}^{2} \frac{\partial^{2}}{\partial x_{i}^{2}}+\sum_{i=1}^{n} \sum_{j>i}^{n}\left(A_{i} A_{j}+A_{j} A_{i}\right) \frac{\partial^{2}}{\partial x_{i} \partial x_{j}}\right) \psi \\
= & \left(-i\left(\sum_{i=1}^{n} A_{i} \frac{\partial}{\partial x_{i}}\right)\left(\sum_{j=1}^{n} q B_{j} V_{j}+m \sigma\right)\right) \psi \\
& -i\left(\sum_{i=1}^{n} q B_{i} V_{i}+m \sigma\right)\left(\left(\sum_{j=1}^{n} A_{j} \frac{\partial}{\partial x_{j}}\right) \psi\right)+\sum_{i=1}^{n} A_{i} \frac{\partial J}{\partial x_{i}} \\
= & -i\left(\sum_{i=1}^{n} \sum_{j=1}^{n} q A_{i} B_{j} \frac{\partial V_{j}}{\partial x_{i}}\right) \psi-\left(\sum_{i=1}^{n} \sum_{j>i}^{n} q^{2}\left(B_{i} B_{j}+B_{j} B_{i}\right) V_{i} V_{j}\right. \\
& \left.-2 i \sum_{i=1}^{n} q m B_{i} V_{i} \sigma-m^{2} \sigma^{2}\right) \psi-i\left(\sum_{i=1}^{n} q B_{i} V_{i}+m \sigma\right) J+\sum_{i=1}^{n} A_{i} \frac{\partial J}{\partial x_{i}}
\end{aligned}
$$

Dirac equation for an arbitrary field can be formulated from the system of linear first order partial differential equations given in Equation (9) by setting $B_{i}=A_{i}=\gamma_{i}, \sigma=1, J=0$ and $A_{i} A_{j}+A_{j} A_{i}=0$. In this case, in terms of the operator $\gamma_{i}$, Equation (9) becomes

$$
\left(\sum_{i=1}^{4} \gamma_{i} \frac{\partial}{\partial x_{i}}\right) \psi=-i\left(\sum_{i=1}^{4} q \gamma_{i} V_{i}+m\right) \psi
$$

Equation (12) can be written in a covariant form as Dirac equation for an arbitrary field as

$$
\left(\gamma^{\mu}\left(i \partial_{\mu}-q V_{\mu}\right)-m\right) \psi=0
$$

Equation (11) also reduces to the following equation

$$
\left(\sum_{i=1}^{4} \gamma_{i}^{2} \frac{\partial^{2}}{\partial x_{i}^{2}}\right) \psi=\left(-i \sum_{i=1}^{4} \sum_{j>i}^{4} q \gamma_{i} \gamma_{j}\left(\frac{\partial V_{j}}{\partial x_{i}}-\frac{\partial V_{i}}{\partial x_{j}}\right)+2 i \sum_{i=1}^{4} q m \gamma_{i} V_{i}-m^{2}\right) \psi
$$

Even though in the following we will examine only physical states of Dirac quantum particles in which $V_{i}=0$ where the physical quantity $V_{i}$ is assumed to be associated with an external field, however, if we consider quantum particles as differentiable manifolds which are formed by mass points joined together by 
contact forces then we may suggest that they are endowed with intrinsic geometric and topological structures and in this case the quantity $V_{i}$ may be considered as an internal field that is responsible for the stability of the physical structure of a quantum particle. As we will show below this is in fact the case when at least part of a quantum particle exists as a two-dimensional structure in which the intrinsic angular momentum can take half-integer values. The problem that we considered can actually be started with Dirac equation given in Equation (13). It can be shown that in the non-relativistic limit, Dirac equation reduces to the Pauli equation for stationary system as [20]

$$
\left(\frac{1}{2 \mu}(-i \hbar \nabla-q \mathbf{A})^{2}-\frac{q \hbar}{2 \mu}(\boldsymbol{\sigma} \cdot \mathbf{B})+q \phi\right) \psi(\mathbf{r})=E \psi(\mathbf{r})
$$

In the case when $\mathbf{A}=0, \mathbf{B}=0$, and $q \phi=k / r$ then we have

$$
-\frac{\hbar^{2}}{2 \mu} \nabla^{2} \psi(\mathbf{r})-\frac{k}{r} \psi(\mathbf{r})=E \psi(\mathbf{r})
$$

Now let us examine a physical system that is described by the Schrödinger wave equation given in Equation (16) from the viewpoint of an observer who sees it as a planar system [21] [22] [23]. It is shown that if we consider physical systems whose configuration space is multiply connected, such as the physical system of a hydrogen-like atom in two rather than three dimensions, then multivalued wavefunctions can be used to describe the system [24]. In two-dimensional space, the Schrödinger equation in the planar polar coordinates takes the form

$$
-\frac{\hbar^{2}}{2 \mu}\left[\frac{1}{r} \frac{\partial}{\partial r}\left(r \frac{\partial}{\partial r}\right)+\frac{1}{r^{2}} \frac{\partial^{2}}{\partial \phi^{2}}\right] \psi(r, \phi)-\frac{k}{r} \psi(r, \phi)=E \psi(r, \phi)
$$

Solutions of the form $\psi(r, \phi)=R(r) \Phi(\phi)$ then reduce the above equation to two separate equations for the functions $\Phi$ and $R$

$$
\begin{gathered}
\frac{\mathrm{d}^{2} \Phi}{\mathrm{d} \phi^{2}}+m^{2} \Phi=0 \\
\frac{\mathrm{d}^{2} R}{\mathrm{~d} r^{2}}+\frac{1}{r} \frac{\mathrm{d} R}{\mathrm{~d} r}-\frac{m^{2}}{r^{2}} R+\frac{2 \mu}{\hbar^{2}}\left(\frac{k}{r}-E\right) R=0
\end{gathered}
$$

where $m$ is identified as the angular momentum of the system. From the system of ordinary differential equations given in Equations (18) and (19), the energy spectrum can be found as

$$
E=-\frac{k^{2} \mu}{2 \hbar^{2}(n+m+1 / 2)^{2}}
$$

It is seen that if the physical system is the Bohr model of two-dimensional hydrogen-like atom then the angular momentum $m$ must take half-integral values. Hence, the topological structure of a configuration space of a physical system can determine the quantum nature of an observable of the system. This result should be expected in quantum mechanics since we know that the quantum behaviour of a particle depends almost entirely on the configuration of an experi- 
ment. If, in a particular experiment, the electron of a hydrogen-like atom is constrained to move in a plane, then the orbital angular momentum of the electron must take half-integral values if we use the Schrödinger equation to study the dynamics of the electron and want to retain the same energy spectrum as the Bohr model. As a consequence, it might seem possible to invoke the result to explain the Stern-Gerlach experiment without the necessity of introduction of spin into the quantum theory.

\section{Dirac Real Equation}

In this section we show that Dirac equation for a free particle can be used to describe the state of a fluid of the quantum particle formulated in the theory of classical fluids. For free Dirac quantum particles, Equation (13) reduces to

$$
\left(i \gamma^{\mu} \partial_{\mu}-m\right) \psi=0
$$

By expanding Equation (21) using the matrices $\gamma_{i}$ given in Equation (6), we obtain

$$
\begin{aligned}
& -\frac{\partial \psi_{1}}{\partial t}-i m \psi_{1}=\left(\frac{\partial}{\partial x}-i \frac{\partial}{\partial y}\right) \psi_{4}+\frac{\partial \psi_{3}}{\partial z} \\
& -\frac{\partial \psi_{2}}{\partial t}-i m \psi_{2}=\left(\frac{\partial}{\partial x}+i \frac{\partial}{\partial y}\right) \psi_{3}-\frac{\partial \psi_{4}}{\partial z} \\
& \frac{\partial \psi_{3}}{\partial t}-i m \psi_{3}=\left(-\frac{\partial}{\partial x}+i \frac{\partial}{\partial y}\right) \psi_{2}-\frac{\partial \psi_{1}}{\partial z} \\
& \frac{\partial \psi_{4}}{\partial t}-i m \psi_{4}=\left(-\frac{\partial}{\partial x}-i \frac{\partial}{\partial y}\right) \psi_{1}+\frac{\partial \psi_{2}}{\partial z}
\end{aligned}
$$

First, it is observed that with the form of the field equations given in Equations (22)-(25), we may interpret that the change of the field $\left(\psi_{1}, \psi_{2}\right)$ with respect to time generates the field $\left(\psi_{3}, \psi_{4}\right)$, similar to the case of Maxwell field equations in which the change of the electric field generates the magnetic field. With this observation it may be suggested that, like the Maxwell electromagnetic field which is composed of two essentially different physical fields, the Dirac field of massive particles may also be viewed as being composed of two different physical fields, namely the field $\left(\psi_{1}, \psi_{2}\right)$, which plays the role of the electric field in Maxwell field equations, and the field $\left(\psi_{3}, \psi_{4}\right)$, which plays the role of the magnetic field. The similarity between Maxwell field equations and Dirac field equations can be carried further by showing that it is possible to reformulate Dirac equation as a system of real equations. When we formulate Maxwell field equations from a system of linear first order partial differential equations we rewrite the original Maxwell field equations from a vector form to a system of first order partial differential equations by equating the corresponding terms of the vectorial equations [9]. Now, since, in principle, a complex quantity is equivalent to a vector quantity therefore in order to form a system of real equations from Dirac complex field equations we equate the real parts with the real 
parts and the imaginary parts with the imaginary parts. In this case Dirac equation given in Equations (22)-(25) can be rewritten as a system of real equations as follows

$$
\begin{gathered}
-\frac{\partial \psi_{1}}{\partial t}=\frac{\partial \psi_{4}}{\partial x}+\frac{\partial \psi_{3}}{\partial z} \\
-\frac{\partial \psi_{2}}{\partial t}=\frac{\partial \psi_{3}}{\partial x}-\frac{\partial \psi_{4}}{\partial z} \\
-\frac{\partial \psi_{3}}{\partial t}=\frac{\partial \psi_{2}}{\partial x}+\frac{\partial \psi_{1}}{\partial z} \\
-\frac{\partial \psi_{4}}{\partial t}=\frac{\partial \psi_{1}}{\partial x}-\frac{\partial \psi_{2}}{\partial z} \\
\frac{\partial \psi_{4}}{\partial y}=m \psi_{1} \\
\frac{\partial \psi_{3}}{\partial y}=-m \psi_{2} \\
\frac{\partial \psi_{2}}{\partial y}=-m \psi_{3} \\
\frac{\partial \psi_{1}}{\partial y}=m \psi_{4}
\end{gathered}
$$

If the wavefunction $\psi$ satisfies Dirac field equations given in Equations (26)-(33) then we can derive the following system of equations for all components

$$
\begin{gathered}
\frac{\partial^{2} \psi_{i}}{\partial^{2} y}-m^{2} \psi_{i}=0 \\
\frac{\partial^{2} \psi_{i}}{\partial t^{2}}-\frac{\partial^{2} \psi_{i}}{\partial x^{2}}-\frac{\partial^{2} \psi_{i}}{\partial z^{2}}=0
\end{gathered}
$$

Solutions to Equation (34) are

$$
\psi_{i}=c_{1 i}(x, z) \mathrm{e}^{m y}+c_{2 i}(x, z) \mathrm{e}^{-m y}
$$

where $c_{1 i}$ and $c_{2 i}$ are undetermined functions of $(x, z)$. The solutions given in Equation (36) give a distribution of a physical quantity along the $y$-axis. On the other hand, Equation (35) can be used to describe the dynamics, for example, of a vibrating membrane in the $(x, z)$-plane. If the membrane is a circular membrane of radius a then the domain $D$ is given as $D=\left\{x^{2}+z^{2}<a^{2}\right\}$. In the polar coordinates given in terms of the Cartesian coordinates $(x, z)$ as $x=r \cos \theta, z=r \sin \theta$, the two-dimensional wave equation given in Equation (35) becomes

$$
\frac{1}{c^{2}} \frac{\partial^{2} \psi}{\partial t^{2}}-\frac{\partial^{2} \psi}{\partial r^{2}}-\frac{1}{r} \frac{\partial \psi}{\partial r}-\frac{1}{r^{2}} \frac{\partial^{2} \psi}{\partial \theta^{2}}=0
$$

The general solution to Equation (37) for the vibrating circular membrane with the condition $\psi=0$ on the boundary of $D$ can be found as [6] [11] 


$$
\begin{aligned}
\psi(r, \theta, t)= & \sum_{m=1}^{\infty} J_{0}\left(\sqrt{\lambda_{0 m}} r\right)\left(C_{0 m} \cos \sqrt{\lambda_{0 m}} c t+D_{0 m} \sin \sqrt{\lambda_{0 m}} c t\right) \\
& +\sum_{m, n=1}^{\infty} J_{n}\left(\sqrt{\lambda_{n m}} r\right)\left(A_{n m} \cos n \theta+B_{n m} \sin n \theta\right) \\
& \times\left(\left(C_{n m} \cos \sqrt{\lambda_{n m}} c t+D_{n m} \sin \sqrt{\lambda_{n m}} c t\right)\right)
\end{aligned}
$$

where $J_{n}\left(\sqrt{\lambda_{n m}} r\right)$ is the Bessel function of order $n$ and the quantities $A_{n m}$, $B_{n m}, C_{n m}$ and $D_{n m}$ can be specified by the initial and boundary conditions. It is also observed that at each moment of time the vibrating membrane appears as a $2 \mathrm{D}$ differentiable manifold which is a geometric object whose geometric structure can be constructed using the wavefunction given in Equation (38) as

$$
R=\left(2\left(\psi_{11} \psi_{22}-\left(\psi_{12}\right)^{2}\right) /\left(1+\psi_{1}^{2}+\psi_{2}^{2}\right)^{2}\right.
$$

where $\psi_{\mu}=\partial \psi / \partial x^{\mu}$ and $\psi_{\mu v}=\partial^{2} \psi / \partial x^{\mu} \partial x^{\nu} \quad$ [25]. Even though elementary particles may have the geometric and topological structures of a $3 \mathrm{D}$ differentiable manifold, it is seen from the above descriptions via the Schrödinger wave equation and Dirac equation that they appear as 3D physical objects that embedded in three-dimensional Euclidean space. Interestingly, in the following we will show that the solution given in Equation (38) can be used to describe a standing wave in a fluid due to the motion of two waves in opposite directions. For a steady state in which the system is time-independent, the system of equations given in Equations (26)-(29) reduces to the following system of equations

$$
\begin{aligned}
& \frac{\partial \psi_{2}}{\partial x}+\frac{\partial \psi_{1}}{\partial z}=0, \frac{\partial \psi_{1}}{\partial x}-\frac{\partial \psi_{2}}{\partial z}=0 \\
& \frac{\partial \psi_{4}}{\partial x}+\frac{\partial \psi_{3}}{\partial z}=0, \frac{\partial \psi_{3}}{\partial x}-\frac{\partial \psi_{4}}{\partial z}=0
\end{aligned}
$$

It is observed from Dirac equation for steady states that the field $\left(\psi_{1}, \psi_{2}\right)$ and the field $\left(\psi_{3}, \psi_{4}\right)$ satisfy the Cauchy-Riemann equations in the $(x, z)$-plane. We will now discuss whether it is possible to consider Dirac quantum particles as physical systems which exist in a fluid state as defined in the classical fluid dynamics as substances that retain a definite volume, have the ability to flow and deform continually, hence they can exhibit a wave motion. For references in the next section we will outline the main features in the theory of classical fluids, especially, in two dimensions.

\section{Fluid Dynamics in Two-Dimensions}

In fluid dynamics, quantities that satisfy the Cauchy-Riemann equations can be identified with the velocity potential and the stream function of an incompressible and irrotational flow [26] [27] [28] [29]. In two-dimensional fluid dynamics, a streamline is a theoretical line that is assumed to be tangential to the instantaneous velocity, therefore there is no flow that can cross the streamline. For a continuous stream of fluid, the streamlines can form continuous lines or closed curves. As an illustration, in the following we will consider a free or potential 
vortex flow whose streamlines are concentric circles in the $(x, z)$-plane as shown in Figure 2.

In two-dimensional incompressible flow, the stream function $\Psi$ is defined as a volume flux through a curve given by

$$
\Psi=\int\left(v_{x} \mathrm{~d} z-v_{z} \mathrm{~d} x\right)
$$

From the definition given in Equation (41), we have $\mathrm{d} \Psi=v_{x} \mathrm{~d} z-v_{z} \mathrm{~d} x$. On the other hand, the total derivative of the stream function $\Psi$ is given by $\mathrm{d} \Psi=(\partial \Psi / \partial x) \mathrm{d} x+(\partial \Psi / \partial z) \mathrm{d} z$, therefore we obtain the following relationships between the velocity components $\left(v_{x}, v_{z}\right)$ and the stream function $\Psi$

$$
v_{x}=\frac{\partial \Psi}{\partial z}, v_{z}=-\frac{\partial \Psi}{\partial x}
$$

If the stream function $\Psi$ is defined in terms of polar coordinates $(r, \theta)$ as $\Psi=\Psi(r, \theta)$, then we have $\mathrm{d} \Psi=(\partial \Psi / \partial r) \mathrm{d} r+(\partial \Psi / \partial \theta) \mathrm{d} \theta$. As shown in Figure 3, we also have $\mathrm{d} \Psi=v_{r}(r \mathrm{~d} \theta)-v_{\theta} \mathrm{d} r$.

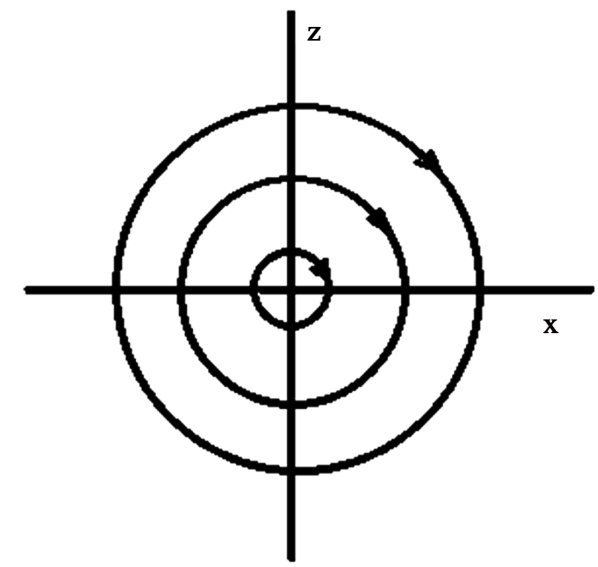

Figure 2. Circular streamlines.

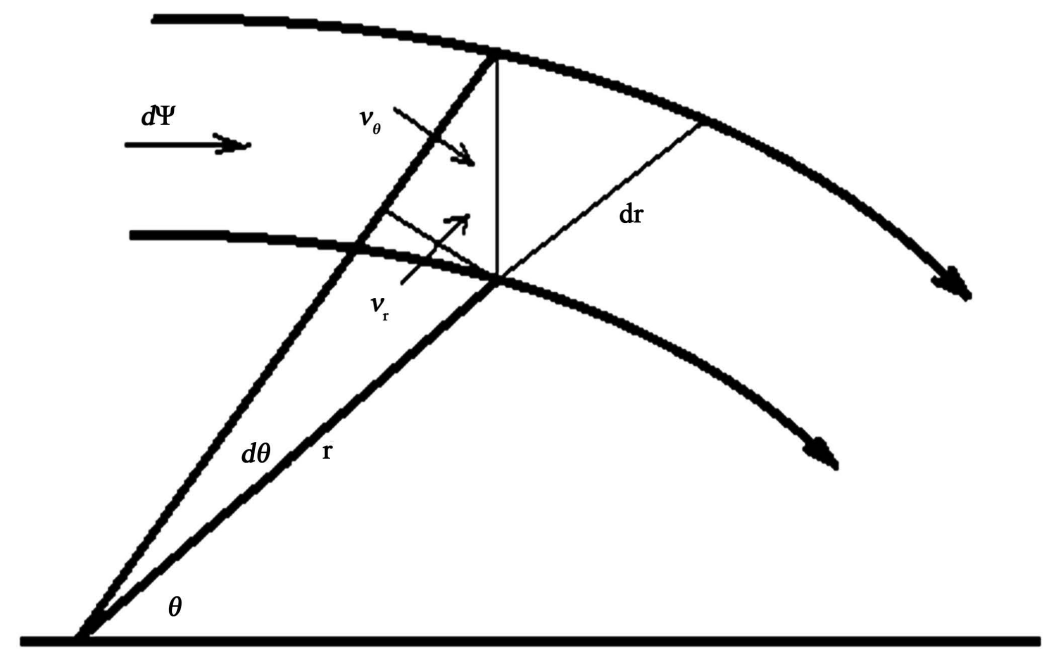

$\mathrm{O}$

Figure 3. Fluid flow in terms of stream function in polar coordinates. 
Therefore we also obtain the following relationships between the velocity components $\left(v_{r}, v_{\theta}\right)$ and the stream function $\Psi$

$$
v_{r}=\frac{1}{r} \frac{\partial \Psi}{\partial \theta}, v_{\theta}=-\frac{\partial \Psi}{\partial r}
$$

From the definition of the stream function we see that the radial component of the velocity of a vortex flow is equals to zero, $v_{r}=0$, since there is no flow that can cross the streamlines. In fluid dynamics, the circulation $\Gamma$ around a closed curve is a line integral of velocity $v$ defined as

$$
\Gamma=\oint \boldsymbol{v} \cdot \mathrm{d} \boldsymbol{s}=\oint v_{s} \mathrm{~d} s
$$

where $v_{s}$ is the tangential velocity. By Stokes' theorem, the circulation $\Gamma$ is related to the vorticity $\omega=\nabla \times \boldsymbol{v}$ as

$$
\Gamma=\oint_{\partial S} v \cdot \mathrm{d} s=\iint_{S} \omega \cdot \mathrm{d} S
$$

It is seen from the above equation that the flux of vorticity is the circulation. In particular, for a two-dimensional flow in the $(x, z)$-plane, the circulation becomes

$$
\Gamma=\int_{\mathrm{A}}\left(\frac{\partial v_{z}}{\partial x}-\frac{\partial v_{x}}{\partial z}\right) \mathrm{d} x \mathrm{~d} z
$$

A flow for which the circulation is equal to zero, $\omega=\nabla \times \boldsymbol{v}=0$, is called a potential or irrotational flow. In two dimensions it is seen from Equation (46) that the condition for potential flow is

$$
\frac{\partial v_{z}}{\partial x}-\frac{\partial v_{x}}{\partial z}=0
$$

It should be mentioned that in potential flow we have $\Gamma=\oint_{\partial S} v \cdot \mathrm{d} \boldsymbol{s}=0$ therefore closed streamlines cannot exist in such flow. In general this result prevents us from identifying the components of Dirac equation $\psi_{1}$ as closed stream function at the same time identifying $\psi_{2}$ as velocity potential. However, it is seen that the result of $\Gamma=\oint v \cdot \mathrm{d} s=0$ may not be valid if the region of space is multiply-connected since the velocity circulation may not be zero if the closed contour cannot be contracted to a point. This important feature can be discussed further as follows. For a two-dimensional irrotational flow given in polar coordinates, it can be shown that the flow velocity $v_{\theta}$ and the radius $r$ satisfy the following relationship $r v_{\theta}=C$, where $C$ is a constant. The constant $C$ can be established by using the singularity in the irrotational vortex flow where to velocity becomes infinite at the centre of the vortex with the vorticity is given by the relation $\omega=-\left(\partial v_{\theta} / \partial r+v_{\theta} / r\right)$. In this case the circulation around a circular streamline can be found as

$$
\Gamma=\oint \boldsymbol{v} \cdot \mathrm{d} \boldsymbol{s}=\oint v_{s} \mathrm{~d} s=2 \pi r v_{\theta}
$$

It is also interesting to note that in the Bohr model of the hydrogen atom in which the electron is assumed to move around the nucleus in stationary circular orbits with $v_{\theta}=v$ then the angular momentum is quantised as $m r v=n \hbar$. If 
we now also assume that Dirac quantum particles are in fluid states whose circulation $\Gamma$ is also quantised as the angular momentum then we obtain the following quantisation for the circulation

$$
\Gamma=2 \pi r v=\frac{2 \pi n \hbar}{m}=\frac{n h}{m}
$$

Since $v_{r}=0$, the stream function $\Psi=\Psi(r, \theta)$ can be obtained as follows

$$
\Psi=-\int v_{\theta} \mathrm{d} r=-\int \frac{\Gamma}{2 \pi r} \mathrm{~d} r=-\frac{\Gamma}{2 \pi} \ln \left(k_{1} r\right)=-\frac{n \hbar}{m} \ln \left(k_{1} r\right)
$$

where $k_{1}$ is an undetermined constant.

In fluid dynamics, another important concept that is connected with an irrotational flow is the concept of the velocity potential $\Phi$ which is defined in the $(x, z)$-plane as

$$
\Phi=\int v_{s} \mathrm{~d} s=\int v_{x} \mathrm{~d} x+v_{z} \mathrm{~d} z
$$

It is seen from Equation (51) that the velocity components can be expressed in terms of the velocity potential as follows

$$
v_{x}=\frac{\partial \Phi}{\partial x}, v_{z}=\frac{\partial \Phi}{\partial z}
$$

In polar coordinates $(r, \theta)$, the velocity potential and its relationship with the velocity components are given as

$$
\begin{gathered}
\Phi=\int v_{s} \mathrm{~d} s=\int v_{r} \mathrm{~d} r+v_{\theta} r \mathrm{~d} \theta \\
v_{r}=\frac{\partial \Phi}{\partial r}, v_{\theta}=\frac{1}{r} \frac{\partial \Phi}{\partial \theta}
\end{gathered}
$$

Similarly, the velocity potential $\Phi$ can also be obtained using the relation $\mathrm{d} \Phi=r v_{\theta} \mathrm{d} \theta+v_{r} \mathrm{~d} r$ as

$$
\Phi=\int r v_{\theta} \mathrm{d} \theta=\int r\left(\frac{\Gamma}{2 \pi r}\right) \mathrm{d} \theta=\int r\left(\frac{n \hbar}{m r}\right) \mathrm{d} \theta=\frac{n \hbar}{m} \theta+k_{2}
$$

where $k_{2}$ is an undetermined constant. From the relationships given in Equations (42) and (52) we then obtain the Cauchy-Riemann equations

$$
\frac{\partial \Phi}{\partial x}+\frac{\partial \Psi}{\partial z}=0, \frac{\partial \Psi}{\partial x}-\frac{\partial \Phi}{\partial z}=0
$$

\section{Fluid state of Dirac Quantum Particles}

By comparing Equation (56) to Dirac equations given in Equations (39) and (40), the field $\left(\psi_{1}, \psi_{2}\right)$ may be identified as the stream function and the velocity potential of one fluid flow and the field $\left(\psi_{3}, \psi_{4}\right)$ with another fluid flow. However, the main problem that we want to deal with now is whether the two fields $\left(\psi_{1}, \psi_{2}\right)$ and $\left(\psi_{3}, \psi_{4}\right)$ are connected and, most importantly, how such connection would lead to the prospect of using them to describe a Dirac quantum particle as a standing wave. In the following we will show that in fact this is the case by using the relationships between the components of these two fields 
given in Equations (30)-(33). For convenience we rewrite these equations as follows

$$
\begin{gathered}
\frac{\partial \psi_{4}}{\partial y}=m \psi_{1}, \frac{\partial \psi_{1}}{\partial y}=m \psi_{4} \\
\frac{\partial \psi_{3}}{\partial y}=-m \psi_{2}, \frac{\partial \psi_{2}}{\partial y}=-m \psi_{3} \\
\frac{\partial^{2} \psi_{i}}{\partial^{2} y}-m^{2} \psi_{i}=0 \text { for } i=1,2,3,4
\end{gathered}
$$

If the physical quantity $m$, which is identified with the inertial mass of a quantum particle, is assumed to be positive, $m>0$, then it is observed that it is possible to describe the physical structure of a Dirac quantum particle as a spinning top if we consider solutions to Equation (59) as hybrid functions of the form

$$
\psi_{i}= \begin{cases}c_{1 i}(x, z) \mathrm{e}^{m y} & \text { for } y<0 \\ c_{2 i}(x, z) \mathrm{e}^{-m y} & \text { for } y \geq 0\end{cases}
$$

For simplicity, instead of the hybrid form given in Equation (60), in the following we will show only for the case in which $y \geq 0$ since similar results can be obtained for which $y<0$. The solutions given in Equation (60) can be rewritten in the following forms

$$
\begin{aligned}
& \psi_{1}=c_{21}(x, z) \mathrm{e}^{-m y}, \psi_{2}=c_{22}(x, z) \mathrm{e}^{-m y} \\
& \psi_{3}=c_{23}(x, z) \mathrm{e}^{-m y}, \psi_{4}=c_{24}(x, z) \mathrm{e}^{-m y}
\end{aligned}
$$

Using the equations given in Equations (57) and (58), we further obtain the conditions $c_{24}=-c_{21}$ and $c_{23}=c_{22}$. If we write $c_{21}=f(x, z)$ and $c_{22}=g(x, z)$ then we have

$$
\begin{aligned}
& \psi_{1}=f(x, z) \mathrm{e}^{-m y}, \psi_{2}=g(x, z) \mathrm{e}^{-m y} \\
& \psi_{3}=g(x, z) \mathrm{e}^{-m y}, \psi_{4}=-f(x, z) \mathrm{e}^{-m y}
\end{aligned}
$$

From the above forms of solutions given to the components $\psi_{i}$ of the wavefunction $\psi$ we can show how a standing wave can be established from the superposition of a wave associated with the field $\left(\psi_{1}, \psi_{2}\right)$ and a wave associated with the field $\left(\psi_{3}, \psi_{4}\right)$. Let $\psi_{1}=f(x, z) \mathrm{e}^{-m y}$ be identified with the velocity potential and $\psi_{2}=g(x, z) \mathrm{e}^{-m y}$ with the stream function of one fluid flow. Now we have two different descriptions that can be given to the field $\left(\psi_{3}, \psi_{4}\right)$. If we identify the component $\psi_{3}=g(x, z) \mathrm{e}^{-m y}$ with the velocity potential and $\psi_{4}=-f(x, z) \mathrm{e}^{-m y}$ with the stream function of another fluid flow then we have the stream function of the first flow equals the velocity potential of the second flow, and the stream function of the second flow is a reflection of the velocity of the first flow. Even though this kind of identification may be used to describe a particular type of fluid flow of Dirac quantum particles, it does not give rise to the physical structure that we are looking for, that is a standing wave. However, 
if we now identify the component $\psi_{3}=g(x, z) \mathrm{e}^{-m y}$ with the stream function and $\psi_{4}=-f(x, z) \mathrm{e}^{-m y}$ with the velocity potential of the second flow then the two flows are identical except for their flow directions, which are opposite to each other, and in fact this is what we want to obtain because they can form a required standing wave. It is also observed that for a steady motion a Dirac quantum particle does not exhibit a wave motion even though it has the potential to establish a wave within its physical structure. Therefore, without an external disturbance a Dirac quantum particle may be considered as a classical particle defined in classical physics. Furthermore, we may also speculate that the two opposite fluid flows associated with the physical structure of a Dirac quantum particle may be related to the concept of spin-half that is introduced into quantum mechanics.

\section{Conclusion}

In our previous works on spacetime structures of quantum particles, we suggest that all quantum particles are formed from mass points which are joined together by contact forces, which is a consequence of viewing quantum particles as CW-complexes. Being identified with differentiable manifolds, quantum particles therefore should be endowed with geometric and topological structures of differentiable manifolds and their motion should be described as isometric embeddings in higher Euclidean space. In particular, we show that quantum particles may have the geometric and topological structures of a 3D differentiable manifold which can be described as standing waves which are solutions to the Schrödinger wave equation and Dirac equation. In this work we have extended our previous discussions by showing that Dirac equation can be used to describe quantum particles as composite structures that are in a fluid state in which the components of the wavefunction can be identified with the stream function and the velocity potential of a potential flow formulated in the theory of classical fluids. With this fluid composition, physically, Dirac quantum particles can manifest as standing waves which are the result of the superposition of two fluid flows moving in opposite directions. However, for a steady motion, a quantum particle whose physical structure is constructed in terms of Dirac equation does not exhibit a wave motion even though it has the potential to establish a wave within its physical structure. Therefore, if there are no external fields acting on it, a Dirac quantum particle may be considered as a classical particle defined in classical physics. It is also noted from the fact that there are two identical fluid flows in opposite directions within their physical structures, the fluid state model of Dirac quantum particles can be invoked to explain why fermions are spin-half particles as discussed in Section 2.

\section{Acknowledgements}

We would like to thank Vanessa Cheng and Jane Gao of the administration of JMP for their editorial advice during the preparation of this work. 


\section{Conflicts of Interest}

The author declares no conflicts of interest regarding the publication of this paper.

\section{References}

[1] Ho, V.B. (2018) International Journal of Physics, 6, 105-115.

[2] Ho, V.B. (2018) GJSFR-A, 18, 37-58.

[3] Ho, V.B. (2018) International Journal of Physics, 6, 139-146.

[4] Einstein, A. (1952) The Principle of Relativity. Dover Publications, New York.

[5] Hamilton, R.S. (1982) Journal of Differential Geometry, 17, 255-306. https://doi.org/10.4310/jdg/1214436922

[6] Strauss, W.A. (1992) Partial Differential Equation. John Wiley \& Sons, Inc., New York.

[7] Cao, H.D. and Zhu, X.P. (2006) Asian Journal of Mathematics, 10, 165-492. https://doi.org/10.4310/AJM.2006.v10.n2.a2

[8] Ho, V.B. (2017) A Derivation of Dirac Equation from a General System of Linear First Order Partial Differential Equations. Preprint, ResearchGate, viXra $1712.0404 \mathrm{v} 1$.

[9] Ho, V.B. (2018) Formulation of Maxwell Field Equations from a General System of Linear First Order Partial Differential Equations. Preprint, ResearchGate, viXra $1802.0055 \mathrm{v} 1$.

[10] Melshko, S.V. (2005) Methods for Constructing Exact Solutions of Partial Differential Equations. Springer Science \& Business Media, Inc.

[11] Sobolev, S.L. (1964) Partial Differential Equations of Mathematical Physics. Dover Publications, Inc, New York.

[12] Dirac, P.A.M. (1928) Proceedings of the Royal Society A: Mathematical, Physical and Engineering Sciences, 117, 610-624. https://doi.org/10.1098/rspa.1928.0023

[13] Jackson, J.D. (1975) Classical Electrodynamics. John Wiley \& Sons, New York.

[14] Landau, L.D. and Lifshitz, E.M. (1987) The Classical Theory of Fields. Pergamon Press, Sydney.

[15] Ho, V.B. (2018) Topological Transformation of Quantum Dynamics. Preprint, ResearchGate, viXra 1810.0300v1.

[16] Bohr, N. (1913) Philosophical Magazine, 26, 1-25.

[17] De Broglie, L. (1925) Annales de Physique, 10, 22-128.

[18] Lachieze-Rey, M. and Luminet, J.P. (2003) Cosmic Topology.

[19] Ho, V.B. (2018) Formulation of Dirac Equation for an Arbitrary Field from a System of Linear First Order Partial Differential Equations.

[20] Bransden, B.H. and Joachain, C.J. (1986) Physics of Atoms and Molecules. Longman Scientific \& Technical, New York.

[21] Ho, V.B. (1994) Journal of Physics A: Mathematical and General, 27, 6237-6241. https://doi.org/10.1088/0305-4470/27/18/031

[22] Ho, V.B. and Morgan, M.J. (1996) Journal of Physics A: Mathematical and General, 29, 1497. https://doi.org/10.1088/0305-4470/29/7/019

[23] Ho, V.B. (1996) Geometrical and Topological Methods in Classical and Quantum Physics. PhD Thesis, Monash University, Clayton. 
[24] Balachandran, A.P., Marmo, G., Skagerstam, B.S. and Stern, A. (1991) Classical Topology and Quantum States. World Scientific, Singapore.

[25] Kreyszig, E. (1975) Introduction to Differential Geometry and Riemannian Geometry. University of Toronto Press, Toronto.

[26] Landau, L.D. and Lifshitz, E.M. (1989) Fluid Mechanics. Pergamon Press, Sydney.

[27] Douglas, J.F., Gasiorek, J.M. and Swaffield, J.A. (1990) Fluid Mechanics. Longman Scientific \& Technical, New York.

[28] Batchelor, G.K. (1988) An Introduction to Fluid Dynamics. Cambridge University Press, Melbourne.

[29] White, F.M. (2011) Fluid Mechanics. McGraw Hill, New York. 